\title{
On Diffraction by Aperiodic Structures
}

\author{
A. Hof ${ }^{\star}$ \\ IHES, Bures-sur-Yvette, France and Department of Mathematics, ETH-Zürich, Switzerland and \\ Institute for Theoretical Physics, University of Groningen, The Netherlands
}

Received: 29 October 1993 / in revised form: 31 May 1994

\begin{abstract}
This paper gives a rigorous treatment of some aspects of diffraction by aperiodic structures such as quasicrystals. It analyses diffraction in the limit of the infinite system, through an appropriately defined autocorrelation. The main results are a justification of the standard way of calculating the diffraction spectrum of tilings obtained by the projection method and a proof of a variation on a conjecture by Bombieri and Taylor.
\end{abstract}

\section{Introduction}

Diffraction by aperiodic structures has attracted a lot of attention since the discovery of quasicrystals [50] (for references, see Sect. 6). This paper gives a rigorous treatment of diffraction by aperiodic structures. A brief discussion of quasicrystals is necessary before it is possible to state the results.

Quasicrystals are alloys having long-range order without being periodic. These properties are inferred from diffraction experiments. Their diffraction spectrum consists of bright spots (the "Bragg peaks"), which means that their structure has long-range order (or, for short, "is ordered"). On the other hand the diffraction spectrum has a symmetry that cannot occur in three-dimensional periodic structures (the symmetry is "crystallographically forbidden"). The structure of quasicrystals, therefore, is not periodic. This is where quasicrystals differ from crystals: the diffraction spectrum of crystals also consists of bright spots but their structure is periodic.

The structure of quasicrystals can, in first approximation, be modelled by aperiodic tilings like those obtained by the so-called projection method (see Sect. 5), in the following way. Let $X$ be the set of vertices of a tiling generated by the projection method and consider

$$
\mu:=\sum_{x \in X} \delta_{x}
$$

$\star$ Current address: Department of Mathematics and Statistics, McMaster University, Hamilton, Ontario, Canada L8S 4K1. E-mail: hof@math.mcmaster.ca 
where $\delta_{x}$ denotes the Dirac delta function at $x$. Its Fourier transform $\hat{\mu}$ can be calculated (see Sect. 5) and is of the form

$$
\hat{\mu}=\sum_{y \in X^{*}} c_{y} \delta_{y}
$$

where the $c_{y}$ are complex numbers and $X^{*}$ is a countable set that is dense in space. It turns out that for suitable tilings and for a suitable cut-off $\alpha$,

$$
\sum_{y \in X^{*}:\left|c_{y}\right|>\alpha}\left|c_{y}\right|^{2} \delta_{y}
$$

gives a good description of the diffraction spectrum of certain quasicrystals if the terms are interpreted as describing spots with an intensity proportional to $\left|c_{y}\right|^{2}$. This recipe has been used for instance in $[35,36,30,10]$. It is the standard way of calculating the diffraction spectrum of structural models of quasicrystals.

The computation of $\hat{\mu}(1.2)$ is possible because $X$ is an infinite set. It follows from standard references on diffraction $[25,14]$ that diffraction by an infinite system is described by the Fourier transform $\hat{\gamma}$ of the autocorrelation of the infinite system

$$
\gamma:=\lim _{L \rightarrow \infty}(2 L)^{-d} \sum_{x, y \in X \cap[-L, L]^{d}} \delta_{x-y}
$$

(this will be discussed in more detail in Sect. 4). Thus two questions arise: whether the recipe that has been used in the quasicrystal literature $[35,36,30,10]$ gives the correct result for tilings obtained by the projection method and whether there is any relation between $\hat{\mu}$ and $\hat{\gamma}$ in more general situations. This paper answers both questions affirmatively. Theorem 5.4 shows that the discrete part of $\hat{\gamma}$ is given by

$$
\sum_{y \in X^{*}}\left|c_{y}\right|^{2} \delta_{y}
$$

which justifies the recipe used in the quasicrystal literature. More generally, Theorem 3.4 shows that the discrete part of $\hat{\gamma}$ is fully determined by the "Fourier coefficients" of $\mu$ under (physically) fairly mild conditions on the set $X$. The arguments used to prove these results also show that, in tilings obtained by the projection method, every finite configuration of tiles occurs with a well-defined frequency and that the $c_{y}$ in (1.2) define continuous eigenfunctions of an associated dynamical system.

The observation that in the limit of the infinite system diffraction is described by the autocorrelation (1.3) makes it possible to solve another problem about diffraction by aperiodic systems. The discovery of quasicrystals has raised the question which configurations of atoms look ordered in diffraction experiments, in the sense that their diffraction spectrum consists of bright spots. This problem has been addressed in dimension one by Bombieri and Taylor [6,7]. They have stated without proof a condition on $\mu$ under which there should be a delta function at $\xi$ in $\hat{\mu}$. Their work has been fairly influential (for references, see Sect. 6). In Sect. 6 it will be proved that their condition, slightly modified, gives the delta functions in the Fourier transform of the autocorrelation of $\mu$ (instead of in $\hat{\mu}$ ). As noted above, it is the Fourier transform of the autocorrelation that describes diffraction.

The point of view taken in this paper is that the set $X$ of atomic positions is fixed in space. One can, however, as is usual in statistical mechanics, also consider the set of all the translates of $X$ and close it in a topology of hard-sphere particle systems 
(see e.g. [29], Appendix B). This gives a compact space on which the group of translations acts continuously, i.e. a dynamical system. Then the spectrum of translations acting as unitary operators on a suitable Hilbert space (the $L^{2}$-functions with respect to an invariant measure on the compact space) can be related to diffraction. This has been done by Van Enter and Miękisz [19] for systems with $X \subset \mathbb{Z}^{d}$ and $\mathbb{Z}^{d}$-action, and for continuous systems with hard-core condition by Dworkin [18]. These points of view are complementary. The "static" point of view naturally leads to "dynamical" results, like the construction of continuous eigenfunctions from the $c_{y}$.

Several authors (e.g. [46, 43, 5, 42, 44, 51]) have recently studied so-called tiling dynamical systems. These are the dynamical systems that result if one considers the sets of translates of tilings, rather then sets of translates of sets of atomic positions.

The paper is organized as follows. Section 2 introduces and discusses the notion of the autocorrelation. Section 3 contains general results on Fourier transforms of unbounded measures and autocorrelations. These results are used in Sects. 5 and 6. Section 4 discusses why diffraction by infinite systems is described by the autocorrelation defined in Sect. 2 and explains why one should consider diffraction by infinite systems at all. This section can be read (almost) independently of Sects. 2 and 3. Section 5 contains the results on tilings generated by the projection method. Section 6 discusses Bombieri and Taylor's conjecture and gives some references to the literature on diffraction by aperiodic structures.

\section{The Autocorrelation of Unbounded Measures}

This section defines and discusses the notion of the autocorrelation of a measure. Of particular importance for the discussion of diffraction will be the discrete measures described in Example 2.1. For a general reference on measure and integration, see [15]. Below, measures are complex measures unless stated otherwise.

A measure $\mu$ on $\mathbb{R}^{d}$ is a linear functional on the space $\mathscr{K}$ of complex continuous functions on $\mathbb{R}^{d}$ of compact support with the property that for every compact subset $K$ of $\mathbb{R}^{d}$ there is a constant $a_{K}$ such that

$$
|\mu(f)| \leqq a_{K}\|f\|
$$

for all complex bounded functions with support in $K$; here $\|\cdot\|$ denotes the supremum norm. A measure is called positive if $\mu(f) \geqq 0$ for all $f \geqq 0$. For every measure $\mu$ there is a smallest positive measure $\rho$ such that $|\mu(f)| \leqq \rho(|f|)$ for all $f \in \mathscr{K}$ (see e.g. [15], Proposition 13.3.2). The measure $\rho$ is called the absolute value of $\mu$ and will be denoted by $|\mu|$. A measure $\mu$ is called bounded if $|\mu|\left(\mathbb{R}^{d}\right)$ is finite, otherwise it is called unbounded. The set of measures on $\mathbb{R}^{d}$ is given the vague topology: a sequence of measures $\left\{\mu_{n}\right\}$ converges to $\mu$ in the vague topology if $\lim _{n \rightarrow \infty} \mu_{n}(f)=\mu(f)$ for all $f \in \mathscr{K}$.

For any function $f$, define $\breve{f}$ by $\breve{f}(x):=f(-x)$ and $\tilde{f}$ by $\tilde{f}:=\bar{f}$, where the bar denotes complex conjugation. Similarly, for a measure $\mu$, define $\check{\mu}$ by $\breve{\mu}(f):=\mu(\check{f})$ and $\bar{\mu}$ by $\bar{\mu}(f):=\mu(\bar{f})$ and $\tilde{\mu}$ by $\tilde{\mu}:=\bar{\mu}$. Recall that the convolution $\mu * v$ of two measures $\mu$ and $v$ is defined by $\mu * v(f):=\int \mu(d x) v(d y) f(x+y)$; it is welldefined if at least one of the two measures has compact support. For every positive number $L$, let $C_{L}$ denote the closed cube of side $L$ centered around the origin. The characteristic function of a subset $A$ of $\mathbb{R}^{d}$ is denoted by $1_{A}$. 
The restriction of a measure $\mu$ to $C_{L}$ is denoted by $\mu_{L}$. Since $\mu_{L}$ has compact support,

$$
\gamma^{L}:=L^{-d} \mu_{L} * \tilde{\mu}_{L}
$$

is well defined. Every vague limit point of the $\gamma^{L}$ as $L \rightarrow \infty$ is called an autocorrelation of $\mu$. So an autocorrelation is by definition a measure. In this paper, all measures that have an autocorrelation will have just one autocorrelation. It will be denoted by $\gamma$, or by $\gamma_{\mu}$ if the dependence on $\mu$ has to be stressed.

Let us give some examples. If for a function $f$ on $\mathbb{R}$ the limit

$$
c(x):=\lim _{T \rightarrow \infty} \frac{1}{2 T} \int_{-T}^{T} f(x+\xi) \overline{f(\xi)} d \xi
$$

exists for all $x$ then the function $c$ is called the autocorrelation function of $f[52,53]$. It $f$ is also locally $L^{1}$ with respect to the Lebesgue measure $\lambda$ then $f \lambda$ is a measure and the autocorrelation or $f \lambda$ is easily seen to be $c \lambda$ under the additional assumption that $f$ is bounded. For instance, every uniformly almost-periodic function (on $\mathbb{R}$ or $\mathbb{R}^{d}$ ) has a unique autocorrelation (which is itself a uniformly almost-periodic function). This shows that there are many measures that have an autocorrelation. It also explains why we speak of the "autocorrelation" instead of the "autocorrelation measure" or "autocorrelation function". We do not distinguish between a function $c$ and the measure $c \lambda$.

The following example describes a class of discrete measures and their autocorrelations. They will be very important for the discussion of diffraction.

2.1. Example. Let $X$ be a countable subset of $\mathbb{R}^{d}$ that is uniformly locally finite in the sense that for every compact $K \subset \mathbb{R}^{d}$ there exists a constant $a_{K}$ such that the number of points of $X$ in $K+x$ is bounded by $a_{K}$, uniformly in $x$; here $K+x$ denotes the set $\left\{z \in \mathbb{R}^{d} \mid z-x \in K\right\}$. Later, $X$ will be interpreted as a set of atomic positions. Let $\delta_{x}$ denote the Dirac measure at $x$ and consider the measure

$$
\mu:=\sum_{x \in X} \delta_{x}
$$

Let $A$ be the set $\{x-y \mid x, y \in X\}$ of "interatomic vectors." Assume that $A$ is locally finite in the sense that every compact subset of $\mathbb{R}^{d}$ contains finitely many points of $A$. Note that by definition $a \in A$ implies $-a \in A$. For $a \in A$ and positive $L$ let $N_{L}(a)$ be the number of occurrences of $a$ in the cube $C_{L}$ :

$$
N_{L}(a):=\mid\left\{x \in X \mid x \in C_{L} \text { and } x-a \in X \cap C_{L}\right\} \mid \text {. }
$$

Assume that for all $a \in A$ the limit

$$
n_{a}:=\lim _{L \rightarrow \infty} L^{-d} N_{L}(a)
$$

exists and that $n_{a}>0$. Then $\mu$ has a unique autocorrelation $\gamma$ given by

$$
\gamma:=\sum_{a \in A} n_{a} \delta_{a}
$$

This can be seen as follows. We have

$$
\mu_{L} * \tilde{\mu}_{L}=\sum_{x, y \in X \cap C_{L}} \delta_{x-y}=\sum_{a \in A} N_{L}(a) \delta_{a},
$$


since $\mu_{L}=\sum_{x \in X \cap C_{L}} \delta_{x}$ and $\delta_{x} * \tilde{\delta}_{y}=\delta_{x} * \delta_{-y}=\delta_{x-y}$. Now choose $N>0$ and an $f \in \mathscr{K}$ with support in $C_{N}$, so that $f(a)=0$ if $a \notin C_{N}$. Then

$$
\gamma^{L}(f)=L^{-d} \sum_{a \in A: a \in C_{N}} N_{L}(a) \delta_{a}(f) \text {. }
$$

Since the number of terms in the summation is finite, (2.3) gives

$$
\lim _{L \rightarrow \infty} \gamma^{L}(f)=\sum_{a \in A} n_{a} \delta_{a}(f) .
$$

Because $N$ and $f$ were arbitrary, (2.4) is proven.

If $X$ is the set of vertices of a tiling of $\mathbb{R}^{d}$ that is either generated by the projection method (see Sect. 5) or by a "primitive substitution" as described in e.g. [20], then all hypotheses on $X$ and $A$ are satisfied.

A measure is called translation bounded ([1], p. 5) if for every compact set $K \subset \mathbb{R}^{d}$ there is a constant $\alpha_{K}$ such that

$$
\sup _{x \in \mathbb{R}^{d}}|\mu|(K+x) \leqq \alpha_{K}
$$

The measures in Example 2.1 are translation bounded by the assumption that $X$ is uniformly locally finite. Translation boundedness will be needed in the discussion of the Fourier transform of measures. The following proposition shows that the autocorrelations in Example 2.1 are translation bounded. It also implies that every translation bounded measure has at least one autocorrelation, because it shows that the set $\left\{\gamma^{L}\right\}$ is precompact in the vague topology.

2.2. Proposition. If a translation bounded measure $\mu$ has an autocorrelation $\gamma$, then the measures $\left\{\gamma^{L}\right\}_{L=1}^{\infty}$ and $\gamma$ are all translation bounded with constants $\alpha_{K}$ that are independent of $L$.

Proof. Let $K$ be compact subset of $\mathbb{R}^{d}$ and $\alpha_{K}$ a constant satisfying (2.5). Then

$$
\begin{aligned}
\left|\mu_{L} * \tilde{\mu}_{L}\right|(K+x) & \leqq\left|\mu_{L}\right| *\left|\tilde{\mu}_{L}\right|(K+x) \\
& =\int\left|\mu_{L}\right|(d s)\left|\tilde{\mu}_{L}\right|(K+x-s) \\
& \leqq \alpha_{K} \int\left|\mu_{L}\right| \\
& \leqq \alpha_{K} \alpha_{C_{1}} L^{d} .
\end{aligned}
$$

The first inequality uses a general property of the convolution (see e.g. [15], 14.5.2). The last inequality uses the translation boundedness of $\mu$ and the fact that the number of cubes of side 1 needed to cover a cube of side $L$ is $L^{d}$. Hence

$$
\gamma^{L}(K+x) \leqq 2 \alpha_{K} \alpha_{C_{1}} \quad \text { for all } L \geqq 1
$$

for all $x$, which was to be shown.

Different measures can have the same autocorrelation. If a translation bounded measure $\mu$ has a unique autocorrelation $\gamma_{\mu}$, then clearly $\gamma_{\mu}=\gamma_{\mu+v}$ for every bounded measure $v$. The following proposition gives a class of unbounded measures with the property that they can be added to $\mu$ without changing the autocorrelation.

2.3. Proposition. Let $\mu$ be a translation bounded measure that has a unique autocorrelation $\gamma$. Let $D \subset \mathbb{R}^{d}$ be such that

$$
\lim _{L \rightarrow \infty} L^{-d} \lambda\left(D_{r} \cap C_{L}\right)=0 \quad \text { for all } r>0,
$$


where $D_{r}:=\left\{x \in \mathbb{R}^{d} \mid \operatorname{dist}(x, D)<r\right\}$ and $\lambda$ denotes the Lebesgue measure. Let $v$ be a translation bounded measure such that $v(A)=0$ if $A \cap D=\emptyset$, for all compact $A \subset \mathbb{R}^{d}$. Then $\gamma_{\mu}=\gamma_{\mu+v}$.

Proof. By the linearity of the convolution product, it suffices to show that for every $\phi \in \mathscr{K}$ such that $\phi \geqq 0$,

$$
\lim _{L \rightarrow \infty} L^{-d} \mu_{L} * \tilde{v}_{L}(\phi)=0 \text { and } \lim _{L \rightarrow \infty} L^{-d} v_{L} * \tilde{v}_{L}(\phi)=0
$$

We only prove the first statement, the proof of the second one is similar. We have

$$
\left|\mu_{L} * \tilde{v}_{L}\right|(\phi) \leqq \int\left|\mu_{L}\right|(d s)\left|\tilde{v}_{L}\right|(d t) \phi(s+t) .
$$

Now $\int\left|\tilde{v}_{L}\right|(d t)(s+t)$ is bounded in $s$ since $v$ is translation bounded; it is 0 if $(s-A) \cap D=\emptyset$, where $A$ denotes the support of $\phi$. Therefore, (2.6) and the translation boundedness of $\mu$ imply (2.7).

\section{Measures, Distributions and the Fourier Transform}

This section discusses the Fourier transformation of unbounded measures. It first fixes notation and recalls some definitions. Then it states some results. The two theorems will be used in the discussion of diffraction.

Let $\mathscr{S}$ be the Schwartz space of rapidly decreasing test functions (see Sect. VII.3 in [48]). The Fourier transform $\hat{\phi}$ of $\phi \in \mathscr{S}$ is defined by

$$
\hat{\phi}(\xi):=\int \phi(x) e^{-2 \pi i\langle\xi, x\rangle} d x,
$$

where $\langle\cdot, \cdot\rangle$ denotes the Euclidean inner product in $\mathbb{R}^{d}$. The function $\hat{\phi}$ is itself an element of $\mathscr{S}$. It is sometimes convenient to write $\phi^{\wedge}$ instead of $\hat{\phi}$. One has

$$
\phi(x)=\int \hat{\phi}(\xi) e^{2 \pi i\langle x, \xi\rangle} d \xi
$$

and $\phi^{\wedge}=\check{\phi}$. The relation between the Fourier transform of $\phi$ and that of its translate $\tau_{a} \phi$ defined by $\tau_{a} \phi(x):=\phi(x-a)$ is given by

$$
\widehat{\tau_{a} \phi}(\xi)=e^{-2 \pi i\langle a, \xi\rangle} \hat{\phi}(\xi) \text {. }
$$

A tempered distribution is a continuous linear functional on $\mathscr{S}$ (for the topology on $\mathscr{S}$, see [48]). The Fourier transform $\hat{T}$ of a tempered distribution $T$ is defined by

$$
\hat{T} \phi:=T \hat{\phi} \text { for all } \phi \in \mathscr{S} \text {; }
$$

this is again a tempered distribution. Recall that the Fourier transform of the Dirac measure at $x$ is given by

$$
\hat{\delta}_{x}(\xi)=e^{-2 \pi \imath\langle x, \xi\rangle} .
$$

If a measure $\mu$ defines a tempered distribution $T_{\mu}$ by

$$
T_{\mu} \phi:=\int d \mu \phi \text { for all } \phi \in \mathscr{S},
$$

then the measure is called tempered. A sufficient condition for a measure to be tempered is that it is slowly increasing in the sense that $\int(1+|x|)^{-k}|\mu|(d x)<\infty$ 
for some $k \in \mathbb{N}$ (Theorem VII.VII in [48]). In particular, every translation bounded measure is slowly increasing and therefore tempered. Often, no distinction is made between a measure and the distribution it defines. For instance, we shall write $\hat{\mu}$ for $\widehat{T}_{\mu}$.

The Fourier transform of a tempered measure is a tempered distribution; it may or may not be a measure. For instance, if $\mu$ is positive definite in the sense that $\mu(\phi * \tilde{\phi}) \geqq 0$ for all $\phi \in \mathscr{S}$ then $\hat{\mu}$ is a positive measure by Bochner's Theorem (see e.g. Theorem VII.XVII in [48]). Every autocorrelation is a positive definite. Hence the Fourier transform of an autocorrelation is a positive measure. Examples of measures whose Fourier transforms are not measures will be encountered in Sect. 5.

Theorem 3.2 below shows that if $\hat{\mu}$ is a translation bounded measure then the "Fourier coefficients" of $\mu$ exist uniformly with respect to position; moreover, they determine the discrete part of $\hat{\mu}$. It was derived in an attempt to prove a conjecture by Bombieri and Taylor (see Sect. 6). Theorem 3.4 shows that the discrete part of the Fourier transform of the autocorrelation of a translation bounded measure is uniquely determined by the Fourier coefficients of the measure itself, provided these exist uniformly with respect to position.

3.1. Proposition. Let $\mu$ be a tempered measure. Assume that $\hat{\mu}$ is a measure. Let $\left\{\phi_{n}\right\}_{n=1}^{\infty}$ be a sequence of test functions such that: (i) $\left|\phi_{n}\right| \leqq f$, for all $n$, for some $f \in L^{1}(\hat{\mu})$; (ii) $\phi_{n}(\xi)=1$ for all $n$ and ( iii) $\phi_{n}(t) \rightarrow 0$ as $n \rightarrow \infty$ if $t \neq \xi$. Then

$$
\hat{\mu}(\{\xi\})=\lim _{n \rightarrow \infty} \mu\left(\hat{\phi}_{n}\right) .
$$

Proof. Observe that $\hat{\mu}-\hat{\mu}(\{\xi\}) \delta_{\xi}$ is a measure and that $f \in L^{1}\left(\hat{\mu}-\hat{\mu}(\{\xi\}) \delta_{\xi}\right)$. By the Lebesgue dominated convergence theorem

$$
\left(\hat{\mu}-\hat{\mu}(\{\xi\}) \delta_{\xi}\right)\left(\phi_{n}\right) \rightarrow 0 \text { as } n \rightarrow \infty .
$$

This implies the desired result, since by the definition of the Fourier transform $\hat{\mu}\left(\phi_{n}\right)=\mu\left(\hat{\phi}_{n}\right)$ for all $n$.

3.2. Theorem. Let $\mu$ be a translation bounded measure and suppose that $\hat{\mu}$ is also a translation bounded measure. Then

$$
\hat{\mu}(\{\xi\})=\lim _{n \rightarrow \infty} n^{-d} \int_{C_{n}+a_{n}} e^{-2 \pi i\langle\xi, x\rangle} \mu(d x)
$$

for every $\xi \in \mathbb{R}^{d}$ and every sequence $\left\{a_{n}\right\} \subset \mathbb{R}^{d}$.

Proof. The result would follow from Proposition 3.1 if it could be applied with

$$
\hat{\phi}_{n}(x)=n^{-d} e^{-2 \pi \iota\langle\xi, x\rangle} 1_{C_{n}+a_{n}}(x) ;
$$

so by (3.2) one would have to take

$$
\phi_{n}(y)=n^{-d} e^{-2 \pi \imath\left\langle a_{n}, y\right\rangle} \prod_{k=1}^{d} \frac{\sin \left(\pi n(\xi-y)_{k}\right)}{\pi(\xi-y)_{k}} .
$$

These functions clearly satisfy conditions (ii) and (iii) of Proposition 3.1, but in general not condition (1). However, it is possible to apply Proposition 3.1 to regularizations $\widehat{\psi_{n}^{e}}$ of $\hat{\phi}_{n}$. The translation boundedness will enable us to take the limit $\varepsilon \rightarrow 0$ and prove the theorem. 
We shall prove the theorem for $\xi=0$. The general result then follows from $\hat{\mu}(\{\xi\})=\left(\delta_{\xi} * \hat{\mu}\right)(\{0\})$ and $\left(\delta_{\xi} * \hat{\mu}\right)=\left(e^{-2 \pi i\langle\xi, \bullet\rangle} \mu\right)^{\wedge}$.

Let $\omega$ be a positive, even $C^{\infty}$-function which is zero if $|x|>1$ and which is normalized in the sense that $\int \omega(x) d x=1$ (for a construction of such a function see e.g. page 55 of [16]). For $\varepsilon>0$ define $\omega_{\varepsilon}(x):=\varepsilon^{-d} \omega(x / \varepsilon)$. The functions $\omega_{\varepsilon}$ are also positive, normalized, even $C^{\infty}$-functions and they vanish for $|x|>\varepsilon$. Clearly, $\omega_{\varepsilon} \in \mathscr{S}$ for all $\varepsilon>0$.

Let

$$
\hat{\phi}_{n}(x):=n^{-d} 1_{C_{n}+a_{n}}(x),
$$

and define

$$
\widehat{\psi_{n}^{\varepsilon}}:=\omega_{\varepsilon} * \hat{\phi}_{n}
$$

It can be shown that $\widehat{\psi_{n}^{\varepsilon}} \rightarrow \hat{\phi}_{n}$ as $\varepsilon \rightarrow 0$ Lebesgue almost everywhere, and, moreover, that the convergence is uniform on sets on which $\hat{\phi}_{n}$ is uniformly continuous (see e.g. page 56 in [16]). Note that $\widehat{\psi_{n}^{\varepsilon}} \in \mathscr{S}$.

Let $\partial C_{n}$ denote the boundary of $C_{n}$ and define

$$
\begin{aligned}
& K_{n}:=\left\{x \in C_{n} \mid \operatorname{dist}\left(x, \partial C_{n}+a_{n}\right) \geqq 2\right\}, \\
& K_{n}^{\prime}:=\left\{x \in \mathbb{R}^{d} \mid \operatorname{dist}\left(x, \partial C_{n}+a_{n}\right)<2\right\} .
\end{aligned}
$$

Then for $\varepsilon<1$ we have

$$
\begin{array}{ll}
\widehat{\psi_{n}^{\varepsilon}}(x)=\hat{\phi}_{n}(x)=0, & \text { if } x \notin K_{n} \cup K_{n}^{\prime}, \\
\widehat{\psi_{n}^{\varepsilon}}(x)=\hat{\phi}_{n}(x)=n^{-d}, & \text { if } x \in K_{n}, \\
\left|\psi_{n}^{\varepsilon}(x)-\hat{\phi}_{n}(x)\right| \leqq n^{-d}, & \text { if } x \in K_{n}^{\prime} .
\end{array}
$$

Let us now verify that the $\widehat{\psi_{n}^{\varepsilon}}$ satisfy conditions (i), (ii) and (iii) of Proposition 3.1. Since $\omega_{\varepsilon}$ is even we have

$$
\psi_{n}^{\varepsilon}(y)=\widehat{\omega_{\varepsilon}}(y) e^{2 \pi i\left\langle a_{n}, y\right\rangle} \prod_{k=1}^{d} \frac{\sin n \pi y_{k}}{n \pi y_{k}},
$$

so $\left|\psi_{n}^{\varepsilon}\right| \leqq\left|\widehat{\omega_{\varepsilon}}\right|$. Since $\hat{\mu}$ is translation bounded by assumption and $\widehat{\omega_{\varepsilon}} \in \mathscr{S}$, we have $\frac{\omega_{\varepsilon}}{\omega_{\varepsilon}} L^{1}(\hat{\mu})$. This shows that condition (i) is satisfied with $f=\omega_{\varepsilon}$. Condition (ii) requires that $\widehat{\psi_{n}^{\varepsilon}}(0)=1$ for all $\varepsilon$. This follows from $\widehat{\omega_{\varepsilon}}(0)=\int \omega_{\varepsilon}(x) d x=1$. If $t \neq 0$, then $\left|\psi_{n}^{\varepsilon}\right|$ is bounded by a constant times $n^{-d}$. Thus condition (iii) is satisfied too.

Proposition 3.1 now gives

$$
\hat{\mu}(\{0\})=\lim _{n \rightarrow \infty} \int \widehat{\psi_{n}^{\varepsilon}} d \mu .
$$

By (3.3) we have

$$
\left|\int\left(\widehat{\psi}_{n}^{\varepsilon}-\hat{\phi}_{n}\right) d \mu\right| \leqq n^{-d}|\mu|\left(K_{n}^{\prime}\right)
$$

for all $n$ and all $\varepsilon<1$. Covering $K_{n}^{\prime}$ by cubes of side 4 and using the translation boundedness of $\mu$ one sees that $|\mu|\left(K_{n}^{\prime}\right)$ is bounded by a constant times $n^{d-1}$. Letting $\varepsilon \rightarrow 0$ then proves the theorem.

3.3. Proposition. If $\mu$ is a tempered measure and $\hat{\mu}$ is a positive measure, then $\hat{\mu}$ is translation bounded. 
Proof. Let $A$ be a compact subset of $\mathbb{R}^{d}$ and $a \in \mathbb{R}^{d}$. Since $|\hat{\mu}|=\hat{\mu}$, we have to show that $\hat{\mu}(A+a)$ is bounded in $a$. Let $B:=A \cup\left\{x \in \mathbb{R}^{d} \mid \operatorname{dist}(x, A) \leqq 2\right\}$. Note that $1_{B+a} * \omega \in \mathscr{S}$, where $\omega$ is as in the proof of Theorem 3.2. Thus

$$
\hat{\mu}(A+a) \leqq \hat{\mu}\left(1_{B+a} * \omega\right)=\mu\left(\hat{1}_{B+a} \hat{\omega}\right) \leqq|\mu|\left(\left|\hat{1}_{B} \hat{\omega}\right|\right),
$$

uniformly in $a$, since $\hat{1}_{B+a}=e^{-2 \pi i\langle a \cdot \bullet\rangle} \hat{1}_{B}$.

3.4. Theorem. Let $\mu$ be a translation bounded measure that has a unique autocorrelation $\gamma$ and suppose that for all $\xi \in \mathbb{R}^{d}$,

$$
m_{\xi}:=\lim _{L \rightarrow \infty} L^{-d} \int_{C_{L}+a} e^{-2 \pi i\langle\xi, x\rangle} \mu(d x)
$$

exists uniformly in a. Then

$$
\hat{\gamma}(\{\xi\})=\left|m_{\xi}\right|^{2}
$$

for all $\xi$.

Proof. The proof amounts to justifying an interchange of limits. Fix $\varepsilon>0$. We shall show that $\left.|\hat{\gamma}(\{\xi\})-| m_{\xi}\right|^{2} \mid$ is bounded by a constant times $\varepsilon$.

Recall that $\hat{\gamma}$ is a positive measure since $\gamma$ is positive definite. Hence Proposition 3.3 gives that $\hat{\gamma}$ is translation bounded; Proposition 2.2 gives that $\gamma$ itself is translation bounded. By Theorem 3.2 and (3.4), respectively, there exists an $M^{\prime}$ such that for all $M>M^{\prime}$,

$$
\left|\hat{\gamma}(\{\xi\})-M^{-d} \int_{C_{M}} \gamma(d t) e^{-2 \pi i\langle\xi, t\rangle}\right| \leqq \varepsilon
$$

and

$$
\left|m_{\xi}-M^{-d} \int_{C_{M}+a} \mu(d t) e^{-2 \pi i\langle\xi, t\rangle}\right| \leqq \varepsilon \quad \text { for all } a \in \mathbb{R}^{d}
$$

Recall that $\gamma$ is the vague limit of the measures $\gamma^{L}$ defined in (2.1). Let $\phi_{\eta}$ be a continuous function that is zero outside $C_{M+\eta}$, equals $M^{-d} 1_{C_{M}} e^{-2 \pi i\langle\xi, \bullet\rangle}$ on $C_{M}$ and is bounded by $M^{-d}$. Then $\lim _{L \rightarrow \infty} \gamma^{L}\left(\phi_{\eta}\right)=\gamma\left(\phi_{\eta}\right)$. In combination with (3.5) and Proposition 2.2 this gives that there exists a $K^{\prime}$ such that for all $K>K^{\prime}$,

$$
\left|\hat{\gamma}(\{\xi\})-M^{-d} K^{-d} \int \mu_{K}(d u) \tilde{\mu}_{K}(d v) 1_{C_{M}}(u+v) e^{-2 \pi i\langle\xi, u+v\rangle}\right| \leqq 2 \varepsilon .
$$

Take $K^{\prime}>M$. The second term in the left-hand side equals

$$
K^{-d} \int 1_{C_{K}}(u) e^{-2 \pi i\langle\xi, u\rangle} \mu(d u) M^{-d} \int 1_{C_{K}}(u) 1_{C_{M}-u}(v) e^{-2 \pi i\langle\xi, v\rangle} \tilde{\mu}(d v) .
$$

Restricting the first integration from $C_{K}$ to $C_{K-M}$ introduces an error that, by the translation boundedness of $\mu$, is bounded by $\alpha / K$ for some constant $\alpha$. Next, the $1_{C_{K}}$ in the second integration can be dropped. Thus

$$
\left|\hat{\gamma}(\{\xi\})-K^{-d} \int_{C_{K}-M} e^{-2 \pi i\langle\xi, u\rangle} \mu(d u) M^{-d} \int_{C_{M}-u} e^{-2 \pi i\langle\xi, v\rangle} \tilde{\mu}(d v)\right| \leqq 2 \varepsilon+\alpha / K .
$$

By (3.6) and the translation boundedness of $\mu$ we then obtain that there exist a $K^{\prime \prime}$ and a constant $\alpha^{\prime}$ such that for all $K>K^{\prime \prime}$,

$$
\left.|\hat{\gamma}(\{\xi\})-| m_{\xi}\right|^{2} \mid \leqq \alpha^{\prime} \varepsilon,
$$

which finishes the proof. 
Most of this section is based on Sect. 4.3 in [28]. In particular, the proofs of Proposition 3.1 and Theorem 3.2 can be found there. Theorem 3.4 generalizes Proposition 4.3.6 in [28].

Instead of Proposition 3.3 we could have used, in the proof of Theorem 3.4, a result by Argabright and Gil de Lamadrid ([1], Theorem 2.5) that shows that $\hat{\gamma}$ is translation bounded. We have included Proposition 3.3 for convenience of the reader, since these authors use a definition of the Fourier transform that differs from the definition in distribution theory. Gil de Lamadrid and Argabright also have a result ([21], Theorem 11.4; it uses the same definition of the Fourier transform as [1]) closely related to Theorem 3.2; we became aware of this after finishing the proof of Theorem 3.2 .

\section{Diffraction and the Autocorrelation}

This section explains why diffraction by infinite systems is described by the Fourier transform of the autocorrelation, and that it is wrong in principle to describe it by the Fourier transform of the mass density. Examples show that the two descriptions in general give different results.

Texts on diffraction usually discuss diffraction by finite assemblies of atoms and diffraction by crystals, i.e. infinite assemblies of atoms on a lattice. Consider an assembly of $N$ identical structureless atoms at positions $x_{1}, \ldots, x_{N}$ and model it by the bounded measure $\rho=\sum_{j=1}^{N} \delta_{x_{j}}$. If this assembly is irradiated by radiation of wavelength $\lambda$ from the direction $Q_{0}$, then the intensity of radiation scattered elastically into the direction $Q$ is given by

$$
I(q)=\left|\sum_{j=1}^{N} e^{-2 \pi \imath\left(q, x_{J}\right)}\right|^{2}
$$

where $q=\left(Q-Q_{0}\right) / \lambda$ (see e.g. Chapter 5 in [14], or Sect. 2.2 in [25]). The function $I$ is known as the "distribution of scattering power" ([14], p. 108) or as the "diffracted intensity" ([25], p. 16). For a discussion of how $I$, which is a function on $\mathbb{R}^{3}$, gives rise to diffraction spectra, which are two-dimensional pictures, see e.g. Sect. 5.8 in [14].

Note that

$$
I=|\hat{\rho}|^{2}=(\rho * \tilde{\rho})^{\wedge} .
$$

The convolution product $\rho * \tilde{\rho}$ is known as the "Patterson function" - although here it is a measure - and as the "autocorrelation" (see e.g.[25], p. 32). (Note that the autocorrelation as defined in Sect. 2 is zero for any bounded measure!) The sequence $I$ does not converge as $N \rightarrow \infty$, not vaguely to a measure and not even in the sense of distributions, since $\int I(q)[\phi * \tilde{\phi}](q) d q \geqq N \int[\phi * \tilde{\phi}](q) d q$, for every $\phi \in \mathscr{K}$. For infinite systems, therefore, one has to consider $I / N$, the scattering power per atom [28]. This is analogous to the fact that for an infinite statistical mechanical system it only makes sense to speak about the energy per unit volume, or per particle; the total energy is not defined. If the infinite system has a unique autocorrelation $\gamma$ in the sense of Sect. 2 , then $I / N$ converges vaguely to $\hat{\gamma} / \gamma(\{0\})$ as $N \rightarrow \infty$ (observe that $\gamma(\{0\})$ is the particle density).

Recall from Sect. 3 that $\hat{\gamma}$ is a positive measure. Like any measure, $\hat{\gamma}$ can have a discrete part, an absolutely continuous part and a singular continuous part. A discrete 
part (in addition to the point mass at 0 , which is always present) is interpreted as a sign of order, an absolutely continuous part as a sign of disorder, and a purely singular continuous $\hat{\gamma}$ as describing a kind of order between quasiperiodicity and randomness [4, 3] (for other notions of order and their relations, see e.g. [41]). Now $I / N$ is a continuous function (i.e. a purely absolutely continuous measure) for every $N$. Discrete or singular continuous parts only occur in the limit of the infinite system. Again there is an analogy with statistical mechanics: like the partition function (cf. e.g. [47]), the autocorrelation can only have singularities in the limit of the infinite system.

Let us check what $\hat{\gamma}$ looks like for a crystal. Suppose for simplicity that the lattice is $\mathbb{Z}^{3}$. Let $\rho$ be a decoration of the unit cell by $N$ atoms (i.e. suppose that in (4.1) the coordinates of the $x_{j}$ lie between 0 and 1). Then the crystal structure is modelled by

$$
\mu=\rho * \sum_{n \in \mathbb{Z}^{3}} \delta_{n} .
$$

Since the autocorrelation of $\sum_{n \in \mathbb{Z}^{3}} \delta_{n}$ is $\sum_{n \in \mathbb{Z}^{3}} \delta_{n}$,

$$
\gamma=(\rho * \tilde{\rho}) * \sum_{n \in \mathbb{Z}^{3}} \delta_{n}
$$

so that

$$
\hat{\gamma}=I \sum_{n \in \mathbb{Z}^{3}} \delta_{n}
$$

by the Poisson summation formula. This is exactly the expression for the scattering power of a crystal (see e.g. Sect. 6.1 in [14], or Chapter 4 in [25]). The same argument works for an arbitrary lattice $X$, because the autocorrelation of $\sum_{x \in X} \delta_{x}$ is given by $\alpha \sum_{x \in X} \delta_{x}$, where $\alpha$ is the number of lattice points per unit volume. Note that the expression (4.2) for $\hat{\gamma}$ can also be obtained from $\mu$ by replacing the coefficients of the Delta functions in $\hat{\mu}$ by their moduli squared. As stated in the introduction, that is the procedure that is used to calculate the diffraction spectrum of structural models of quasicrystals $[35,36,30,10]$. The next section will justify this procedure for tilings obtained from the projection method (Theorem 5.4).

In general $\hat{\mu}$ and $\hat{\gamma}$ are rather different objects. If $\mu$ is changed to $\mu^{\prime}$ by moving atoms then $\hat{\mu}$ changes. But if not "too many" atoms are moved, i.e. if $\mu$ and $\mu^{\prime}$ differ on a set that is essentially $(d-1)$-dimensional, then $\gamma_{\mu}=\gamma_{\mu^{\prime}}$ by Proposition 2.3. Hence $\hat{\gamma}_{\mu}=\hat{\gamma}_{\mu^{\prime}}$, too. The meaning of this is that "defects" can only be seen in a diffraction experiment if they occur with a positive density.

So far, atoms have been structureless in the sense that they have been modelled by delta functions. There is no loss of generality in this assumption. One can give them structure (i.e. model them by a cloud of electrons) by convoluting the delta functions with a function $f$ describing the electron cloud. The autocorrelation $\gamma$ then changes to $(f * \tilde{f}) * \gamma$ and $\hat{\gamma}$ to $|\hat{f}|^{2} \hat{\gamma}$.

\section{Diffraction and the Projection Method}

The projection method is a method for generating aperiodic tilings of $\mathbb{R}^{d}$. (A tiling of $\mathbb{R}^{d}$ is a countable covering of $\mathbb{R}^{d}$ by closed sets, which are homeomorphic to closed balls, such that the intersection of the interiors of every pair of sets is empty. The sets are called tiles.) Let $X$ be the set of vertices of a tiling generated 
by the projection method, and $\mu=\sum_{x \in X} \delta_{x}$. This section shows that $\mu$ has a unique autocorrelation $\gamma$, and that the discrete part of $\hat{\gamma}$ is uniquely determined by $\hat{\mu}$. It also shows that every finite configuration of tiles occurs with a well-defined frequency, and shows how the discrete part of $\hat{\gamma}$ is related to continuous eigenfunctions of an associated dynamical system. But first the projection method will be described, following $[30,39]$.

Let $E^{\|}$be a $d$-dimensional linear subspace of $\mathbb{R}^{n}$ and let $E^{\perp}$ be its orthogonal complement. Let $\pi^{\|}$and $\pi^{\perp}$ denote the orthogonal projections on $E^{\|}$and $E^{\perp}$, respectively. Write $x=\left(x^{\|}, x^{\perp}\right)$ for the decomposition of $x \in \mathbb{R}^{n}$ on $E^{\|} \oplus E^{\perp}$. For every bounded subset $K$ of $E^{\perp}$, define the strip

$$
S_{K}:=K+E^{\|}=\left\{x \in \mathbb{R}^{d} \mid x^{\perp} \in K\right\},
$$

the set

$$
X_{K}:=\pi^{\|}\left(S_{K} \cap \mathbb{Z}^{n}\right),
$$

which is a discrete subset of $E^{\|}$, and the measure

$$
\mu_{K}:=\sum_{x \in S_{K} \cap \mathbb{Z}^{n}} \delta_{x}
$$

on $E^{\|}$. Note that $\mu_{K}$ is translation bounded. For reasons that will become clear later, assume that the boundary of $K$ (in $E^{\perp}$ ) is contained in a finite number of $(n-d-1)$-dimensional hyperplanes.

Of special interest is the case where $K$ is the projection $\kappa$ on $E^{\perp}$ of the unit cube $\left\{x \in \mathbb{R}^{n} \mid 0 \leqq x_{i} \leqq 1\right\}$, or translates $\kappa+t$ of $\kappa$ by vectors $t \in E^{\perp}$. If $S_{\kappa+t}$ has no point of $\mathbb{Z}^{n}$ on its boundary, then $t$ is called regular; otherwise $t$ is called singular. The set of regular $t$ is of full Lebesgue measure in every bounded set of $E^{\perp}$ [39]. If $t$ is regular then $S_{\kappa+t}$ contains a unique surface consisting of translates of $d$-facets of the unit cube ([39], Remark VI.2.b). The projections of these facets of $E^{\|}$form the tiles of a tiling of $E^{\|}$; the set of vertices of this tiling is $X_{\kappa+t}$. On identifying $E^{\|}$with $\mathbb{R}^{d}$ - as will be done below without warning - it becomes a tiling of $\mathbb{R}^{d}$. This tiling is denoted by $\mathscr{T}_{t}$. The tiling is completely aperiodic (in the sense that it does not coincide with any of its translates) if $E^{\|} \cap \mathbb{Z}^{n}=\{0\}$. Singular values of $t$ give rise to at least two different tilings, but only after a suitable limit procedure $([8,9,33,34])$. Those tilings are called singular tilings.

The local isomorphism class of a tiling $\mathscr{T}$ is the set of all tilings $\mathscr{T}^{\prime}$ such that every finite configuration of tiles that occurs in $\mathscr{T}$ occurs in $\mathscr{T}^{\prime}$ and vice versa. Different regular values of $t$ can give tilings in different local isomorphism classes. But for every tiling $\mathscr{T}$ in the local isomorphism class defined by a regular value of $t$, there exists a sequence of regular $t_{j}$ such that the tilings $\mathscr{T}_{t}$, converge to a translate $\mathscr{T}^{\prime}$ of $\mathscr{T}$ in the sense that for every $R>0$ there is an $N$ such that for all $j>N$ the tilings $\mathscr{T}_{t_{j}}$ coincide with $\mathscr{T}^{\prime}$ within a sphere of radius $R([32,34,33])$.

Formally, $\hat{\mu}_{K}$ can be computed as follows. Observe that $\mu_{K}$ can be written as

$$
\mu_{K}=\int_{E^{\perp}} 1_{S} \sum_{x \in \mathbb{Z}^{n}} \delta_{n}
$$

The function $1_{S}$ can be viewed as the tensor product $1 \otimes 1_{K}$ of the constant function 1 on $E^{\|}$and the function $1_{K}$ on $E^{\perp}$. So its Fourier transform is $\delta_{0} \otimes \hat{1}_{K}$. Since $\left(\sum_{x \in \mathbb{Z}^{n}} \delta_{x}\right)^{\wedge}=\sum_{\lambda \in \mathbb{Z}^{n}} \delta_{\lambda}$ by the Poisson summation formula, one has

$$
\left[\left(1 \otimes 1_{K}\right) \sum_{x \in \mathbb{Z}^{n}} \delta_{x}\right]^{\wedge}=\left(\delta_{0} \otimes \hat{1}_{K}\right) * \sum_{\lambda \in \mathbb{Z}^{n}} \delta_{\lambda} .
$$


If $f$ is a function on $\mathbb{R}^{n}$ that is absolutely integrable, and $g$ is the function on $E^{\|}$ defined by $g:=\int_{E^{\perp}} f d \lambda$, then $\hat{g}\left(\xi^{\|}\right)=\hat{f}\left(\xi^{\|}, 0\right)$. Therefore, $\hat{\mu}_{K}$ is the restriction to $E^{\|}$of (5.2), i.e.

$$
\hat{\mu}_{K}=\sum_{\lambda \in \mathbb{Z}^{n}} \hat{1}_{K}\left(-\lambda^{\perp}\right) \delta_{\lambda \|}
$$

Thus $\hat{\mu}_{K}$ is indeed of the form (1.2), with $X^{*}=\pi^{\|}\left(\mathbb{Z}^{n}\right)$.

Although $\hat{\mu}_{K}$ is a sum of Dirac delta functions, it is not a measure (unless $\mu_{K}$ is periodic to start with), because the coefficients are not locally absolutely summable ([17], p. 166). This is easily seen if $d=1$ and $n=2$ and $K=\left[-\frac{1}{2}, \frac{1}{2}\right]$, so that $\hat{1}_{K}(\xi)=(\sin \pi \xi) / \pi \xi$. If a series is not absolutely convergent, its terms can be rearranged to give any desired limit. Without further information, therefore, the expression (5.3) is meaningless. De Bruijn $[10,11]$ has first pointed out that $\hat{\mu}_{K}$ should be understood as a limit in the sense of distributions of purely discrete measures

$$
m_{k}=\sum_{\xi \in X^{*}} c_{\xi}^{k} \delta_{\xi}
$$

such that $\lim _{k \rightarrow \infty} c_{\xi}^{k}=c_{\xi}$ for all $\xi$. De Bruijn worked with a theory of distributions that is equivalent to one of the Gelfand-Shilov classes. But in the theory of tempered distributions one can also show that $\hat{\mu}_{K}$ is a limit of purely discrete measures ([17], p. 166). One has to regularize $1_{K}$ in $E^{\perp}$ by convoluting it with the function $\omega_{\varepsilon}$ on $E^{\perp}$. Then $1_{K}$ becomes a rapidly decreasing function, the summation in $\hat{\mu}_{K}$ becomes locally absolutely summable, and $\hat{\mu}_{K}$ becomes a measure. This idea is used in the proof of the following theorem.

5.1. Theorem. Let $K$ be a bounded subset of $E^{\perp}$ whose boundary in $E^{\perp}$ is contained in a finite number of $(n-d-1)$-dimensional hyperplanes. Assume that $E^{\|} \cap \mathbb{Z}^{n}=\{0\}$. Let $\mu_{K}$ be as in (5.1). Then

$$
m_{\xi}:=\lim _{L \rightarrow \infty} L^{-d} \int_{C_{L}+a} e^{-2 \pi i\langle\xi \cdot\rangle} d \mu_{K}
$$

exists uniformly with respect to a for all $\xi$.

Proof. Since $\hat{\mu}_{K}$ is not a measure, Theorem 3.2 does not apply. However, Theorem 3.2 does apply to the regularizations of $\mu_{K}$. The hypothesis on the boundary of $K$ and the assumption that $E^{\|} \cap \mathbb{Z}^{n}=\{0\}$ make it possible to prove the theorem from the existence, uniformly with respect to position, of the Fourier coefficients of the regularizations of $\mu_{K}$.

For $\varepsilon<1$, consider on $E^{\perp}$ the functions $\omega_{\varepsilon}$ introduced in the proof of Theorem 3.2 and define $\phi_{\varepsilon}:=1_{K} * \omega_{\varepsilon}$; this is a function on $E^{\perp}$. Define the regularized measure $\mu_{K, \varepsilon}$ by

$$
\mu_{K, \varepsilon}:=\int_{E \perp} \phi_{\varepsilon} \sum_{x \in \mathbb{Z}^{n}} \delta_{x}
$$

This is a measure on $E^{\|}$. Its Fourier transform is a measure given by

$$
\hat{\mu}_{K, \varepsilon}=\sum_{\lambda \in \mathbb{Z}^{n}} \hat{\phi}_{\varepsilon}\left(-\lambda^{\perp}\right) \delta_{\lambda \perp}
$$

To show that $\hat{\mu}_{K, \varepsilon}$ is translation bounded, consider a compact set $A \subset E^{\|}$. The number of points of $\mathbb{Z}^{n} \cap\left(\pi^{\|}\right)^{-1}(A)$ within a distance $r$ of $A$ is bounded. In fact, one can choose a bound that is the same for all translates in $E^{\|}$of $A$. Since $\hat{\phi}_{\varepsilon}$ 
decreases rapidly, $\hat{\mu}_{K, \varepsilon}$ is translation bounded. Hence, Theorem 3.2 can be applied. It gives that

$$
m_{\xi, \varepsilon}=\lim _{L \rightarrow \infty} L^{-d} \int_{C_{L}+a} e^{-2 \pi \imath\langle\xi, \bullet\rangle} d \mu_{K, \varepsilon}
$$

exists uniformly with respect to $a$. Now

$$
\begin{aligned}
d_{L, \varepsilon} & :=L^{-d}\left|\int_{C_{L}+a} e^{-2 \pi i\langle\xi, \bullet\rangle} d \mu_{K}-\int_{C_{L}+a} e^{-2 \pi i\langle\xi, \bullet\rangle} d \mu_{K, \varepsilon}\right| \\
& \leqq L^{-d} \int_{C_{L}+a} d \mu_{B(\varepsilon)},
\end{aligned}
$$

where $B(\varepsilon):=\left\{x \in E^{\perp} \mid \operatorname{dist}(x, \partial K) \leqq 2 \varepsilon\right\}$. Since the boundary $\partial K$ of $K$ in $E^{\perp}$ is contained in finitely many $(n-d-1)$-dimensional hyperplanes, Proposition 5.2 below shows that $\lim _{\varepsilon \rightarrow 0} \lim _{L \rightarrow \infty} d_{L, \varepsilon}=0$, uniformly in $a$.

5.2. Proposition. Suppose that $E^{\|} \cap \mathbb{Z}^{n}=\{0\}$. Let $H$ be a $(n-1)$-dimensional hyperplane in $\mathbb{R}^{n}$ parallel to $E^{\|}$. Let $\varepsilon>0$ and define $H_{\varepsilon}:=\left\{x \in \mathbb{R}^{n} \mid \operatorname{dist}(x, H) \leqq\right.$ $\varepsilon\}$. Let $R>0$ and define $S_{\varepsilon}:=\left\{x \in H_{\varepsilon} \mid \operatorname{dist}\left(x, E^{\|}\right)<R\right\}$. Then the measure

$$
\mu_{\varepsilon}:=\sum_{x \in S_{\varepsilon} \cap \mathbb{Z}^{n}} \delta_{x} \|
$$

on $E^{\|}$satisfies

$$
\lim _{\varepsilon \rightarrow 0} \lim _{L \rightarrow \infty} L^{-d} \int_{C_{L}+a} d \mu_{\varepsilon}=0
$$

uniformly in a.

Proof. Let $X_{\varepsilon}:=\pi^{\|}\left(S_{\varepsilon} \cap \mathbb{Z}^{n}\right)$ and split $X_{\varepsilon}$ into $X_{H}:=\pi^{\|}\left(S_{\varepsilon} \cap \mathbb{Z}^{n} \cap H\right)$ and $X_{\varepsilon}^{\prime}:=$ $X_{\varepsilon} \backslash X_{H}$. Note that

$$
\int_{C_{L}+a} d \mu_{\varepsilon}=\left|X_{\varepsilon} \cap\left(C_{L}+a\right)\right|
$$

First it will be shown that

$$
\lim _{\varepsilon \rightarrow 0} \lim _{L \rightarrow \infty} L^{-d}\left|X_{\varepsilon}^{\prime} \cap\left(C_{L}+a\right)\right|=0
$$

uniformly in $a$, and then that $X_{H}$ is at most a finite set. These two statements imply (5.6).

Let

$$
D_{\varepsilon}:=\min _{x, y \in \mathbb{Z}^{n} \cap\left(S_{\varepsilon} \backslash H\right)}|x-y|
$$

be the minimum distance in $\mathbb{R}^{n}$ between those points of $\mathbb{Z}^{n}$ that lie in $S_{\varepsilon}$ but not in $H$. Then $D_{\varepsilon} \rightarrow \infty$ as $\varepsilon \rightarrow 0$. This can be seen as follows. Suppose $D_{\varepsilon}$ remains bounded as $\varepsilon \rightarrow 0$. Then there are a $D>0$, a sequence $\varepsilon_{i} \rightarrow 0$ and points $x_{l}, y_{l} \in \mathbb{Z}^{n} \cap\left(S_{\varepsilon} \backslash H\right)$ such that $\left|x_{i}-y_{i}\right|<D$ as $\varepsilon \rightarrow 0$. Now $x_{i}-y_{i} \in \mathbb{Z}^{n}$, and the number of points in $\mathbb{Z}^{n}$ within distance $D$ of the origin is finite, so one can suppose that $x_{i}-y_{i}=a$ for some $a \in \mathbb{Z}^{n}$. This implies that $E^{\|}$is parallel to $a$, which contradicts the hypothesis that $E^{\|} \cap \mathbb{Z}^{n}=\{0\}$. The fact that $D_{\varepsilon} \rightarrow \infty$ as $\varepsilon \rightarrow 0$ implies (5.7).

To show that $X_{H}$ is at most a finite set, suppose first that $0 \in H$. Then points of $H$ are of the form

$$
x^{\|}+\sum_{i=1}^{n-d-1} \lambda_{l} v_{i} \quad \lambda_{i} \in \mathbb{R},
$$


where the $v_{l}$ are vectors in $\mathbb{R}^{n}$ such that the vectors $\pi^{\perp}\left(v_{i}\right)$ are independent in $E^{\perp}$. To maximize the number of lattice points in $H$, one should choose all $v_{l} \in \mathbb{Z}^{n}$. Then $H \cap \mathbb{Z}^{n}$ is of the form $\sum_{i=1}^{n-d-1} m_{i} v_{i}$ with $m_{l} \in \mathbb{Z}$. The set $\pi^{\perp}\left(H \cap \mathbb{Z}^{n}\right)$ is discrete in $E^{\perp}$ since the vectors $\pi^{\perp}\left(v_{l}\right)$ are independent in $E^{\perp}$. Because only finitely many points of $H \cap \mathbb{Z}^{n}$ can project on $E^{\perp}$ within a distance $R$ of the origin, $X_{H}$ is finite.

If $0 \notin H$ then there are two possibilities. Either $H \cap \mathbb{Z}^{n}=\emptyset$ and $X_{H}=\emptyset$, or $H \cap \mathbb{Z}^{n}$ is not empty and the argument above shows that $X_{H}$ is finite.

5.3. Remarks 1. The assumption that $E^{\|} \cap \mathbb{Z}^{n}=\{0\}$, which was used only in the Proof of Proposition 5.2, is not essential. Theorem 5.1 and Proposition 5.2 remain true as long as $E^{\|} \cap \mathbb{Z}^{n}$ does not span $E^{\|}$. If $E^{\|} \cap \mathbb{Z}^{n}$ spans $E^{\|}$then the tiling is fully periodic, i.e. periodic in $d$ independent directions, and Theorem 5.1 is trivial. 2. The functions $\phi_{\varepsilon}$ converge pointwise as $\varepsilon \rightarrow 0$. Denote their limit by $\phi$. Now $\phi \neq 1_{K}$; only at the points of continuity of $1_{K}$ does $\phi_{\varepsilon}$ converge to $1_{K}$. For instance, if $K=\kappa$, then $\phi_{\varepsilon} \rightarrow \frac{1}{2}$ in the interiors of the $(n-d-1)$-facets of $\kappa$.

It follows that $\lim _{\varepsilon \rightarrow 0} \mu_{K, \varepsilon}=\mu_{K}$ vaguely if and only if there are no points of $\mathbb{Z}^{n}$ on the boundary of $S_{K}$. If $K=\kappa+t$, that is equivalent to $t$ being regular. But $\mu_{K, \varepsilon}$ converges vaguely as $\varepsilon \rightarrow 0$ regardless of whether or not there are points of $\mathbb{Z}^{n}$ on the boundary of $S_{K}$. Denote that limit by $\mu$ (so $\mu=\mu_{K}$ if there are no points of $\mathbb{Z}^{n}$ on the boundary of $S_{K}$ ). It is $\hat{\mu}$ that has the property that it is a limit in the sense of distributions of purely discrete measures of the purely discrete measures (5.5). Consequently, the distribution $\hat{\mu}_{K}$ (which is not a measure) can have a "continuous part" in the sense that $\mu_{K}-\mu$ is a bounded measure [10,32].

As an example, consider the case $n=2, d=1$, and $K=\kappa$. Let $t$ be singular. Then there are two points of $\mathbb{Z}^{2}$ on the boundary of $S_{\kappa+t}$, one on the "upper" boundary and one on the "lower" boundary. The strip $S_{\kappa+t}$ defines two singular tilings, and all points of $S_{\kappa+t} \cap \mathbb{Z}^{n}$ that do not lie on the boundary of $S_{\kappa+t}$ project on points that are vertices of both singular tilings. If the sets of vertices of the singular tilings are $X$ and $X^{\prime}$, then the measures $\mu_{X}$ and $\mu_{X^{\prime}}$ defined in (1.1) differ by two delta functions. This example is discussed in detail in Sect. 9 of [10] (see also [32]).

Theorem 5.1 implies that finite configurations of tiles occur with well-defined frequencies. Let $t$ be regular. For a fixed finite set of tiles $P$ in $\mathscr{T}_{t}$, let $K_{C_{L}}(P)$ denote the number of copies (i.e. translates) of $P$ in the cube $C_{L}$. Let $p$ be a vertex of one of the tiles in $P$. As is explained in [30], there is a set $M \in E^{\perp}$ (the "acceptance domain"), which is the intersection of finitely many translates in $E^{\perp}$ of $\kappa+t$, such that the points $r$ of $S_{\kappa+t} \cap \mathbb{Z}^{n}$ that project on a point $r^{\|}$at which there is a copy $P^{\prime}$ of $P$ (in the sense that $P^{\prime}=P+a$ and $r^{\|}=p+a$ for some $a \in E^{\|}$) are exactly the points of $S_{\kappa+t} \cap \mathbb{Z}^{n}$ such that $r^{\perp}$ lies in $M$. Since $M$ is the intersection of finitely many translates of $\kappa+t$, it satisfies the hypothesis Theorem 5.1. Therefore, by taking $\xi=0$ in (5.4),

$$
\lim _{L \rightarrow \infty} L^{-d} N_{C_{L}+a}(P)=n_{P}
$$

uniformly in $a$. This means that $P$ occurs in the tilings $\mathscr{T}_{t}$ with a frequency $n_{P}$. Since the convergence is uniform in $a$, the frequency even exists "in the sense of Van Hove" ([20], Theorem 1). The frequency is the same for all tilings in the local isomorphism class of $\mathscr{T}_{t}$, because the configuration of tiles of $\mathscr{T}_{t}$ in $C_{L}$ also occurs in every other tiling in the local isomorphism class. It follows that, if $X$ denotes the set of vertices of $\mathscr{T}_{t}$ and $\mu$ is defined by (1.1), the measure $\mu$ has a unique 
autocorrelation $\gamma$. Moreover, $\gamma$ is the same for all tilings in the local isomorphism class. Theorems 3.4 and 5.1 now give that $\hat{\gamma}(\{\xi\})=\left|m_{\xi}\right|^{2}$ for all $\xi$. The theorem below summarizes these observations.

5.4. Theorem. Let $\mathscr{T}_{t}$ be a tiling defined by a regular value of $t$. Then all tilings in the local isomorphism class of $\mathscr{T}_{t}$ have the same unique autocorrelation $\gamma$ and for all $\xi \in \mathbb{R}^{d}$,

$$
\hat{\gamma}(\{\xi\})=\left|m_{\xi}\right|^{2},
$$

where $m_{\xi}$ is given by (5.4), with $K=\kappa+t$.

5.5. Remarks 1 . Theorem 5.4 shows that the standard method of computing the strengths and positions of the Bragg peaks (taking the moduli squared of the coefficients of the delta functions in (1.2)) gives the correct answer. We have no results on a possible continuous background, i.e. on the continuous part of $\hat{\gamma}$. But we do know that the measure $\hat{\gamma}$ is the same for all tilings in the local isomorphism class, whereas the distribution $\hat{\mu}$ can have a "continuous part" for singular tilings and be "purely discrete" for regular tilings (cf. Remark 5.3.2).

2. Let $\mathscr{C}$ denote the local isomorphism class of a regular tiling. For every tiling $\mathscr{T} \in \mathscr{C}$, consider the measure $\mu(\mathscr{T})$ defined by (1.2). Since Theorem 5.1 holds regardless of whether or not there are points of $\mathbb{Z}^{d}$ on the boundary of the strip, the Fourier coefficients (5.4) exist uniformly with respect to $a$ for all $\mathscr{T} \in \mathscr{C}$. Thus $m_{\xi}$ can be seen as a function on $\mathscr{C}$.

For $\mathscr{T} \in \mathscr{C}$, let $\mathscr{T}_{x}$ denote the tiling obtained by translating $\mathscr{T}$ over $x$. Then $m_{\xi}\left(\mathscr{T}_{x}\right)=e^{-2 \pi i\langle\xi, x\rangle} m_{\xi}(\mathscr{T})$. The set $\mathscr{C}$ can be made into a compact metric space [43] in which a sequence $\left\{\mathscr{T}_{x_{k}}\right\}$ of translates of $\mathscr{T}$ converges to $\mathscr{T}^{\prime}$ if for all $\varepsilon>0$ and all $R>0$ there are an integer $K>0$ and a vector $y \in \mathbb{R}^{d}$ with $|y| \leqq \varepsilon$ such that for all $k>K$ the tilings $\mathscr{T}_{x_{k}-y}$ and $\mathscr{T}^{\prime}$ coincide within a ball of radius $R$. Thus we have an action of $\mathbb{R}^{d}$ on the compact metric space $\mathscr{C}$, and $m_{\xi}$ is an eigenfunction of this "tiling dynamical system." The fact that the Fourier coefficients (5.4) exist uniformly with respect to $a$ implies that the $m_{\xi}$ are continuous eigenfunctions.

\section{Diffraction and Order}

This section explains how our work proves a variation on a conjecture of Bombieri and Taylor $[6,7]$. It also gives references to other work on diffraction by aperiodic structures.

As mentioned in the Introduction, one of the problems raised by the discovery of quasicrystals is which assemblies of atoms look ordered in diffraction experiments $[26,49]$. In view of the discussion in Sect. 4 , this question should be modelled mathematically as follows: a countable set $X \subset \mathbb{R}^{d}$ looks ordered in a diffraction experiment if the measure $\mu$ defined in (1.1) has an autocorrelation $\gamma$ whose Fourier transform $\hat{\gamma}$ is a discrete measure. For physical reasons, one would assume that there is a minimum distance between the points in $X$. Recall that changing $X$ on an essentially $(d-1)$-dimensional set does not effect $\gamma$; one may wish to exclude such changes by imposing a minimality condition (see below).

The question which sets look ordered in a diffraction experiment has been addressed in one dimension by Bombieri and Taylor [6,7]. They, however, say that the set $X$ looks ordered in a diffraction experiment if $\hat{\mu}$ is of the form 


$$
\hat{\mu}=\sum_{\xi \in X^{*}} c_{\xi} \delta_{\xi}+g
$$

where $X^{*}$ is a countable set and $g$ is some function that supposedly models a diffuse background. The starting point of their analysis is the following conjecture: $\hat{\mu}$ has a Dirac delta function at $\xi$ if and only if

$$
\lim _{N \rightarrow \infty} N^{-1} \sum_{x \in \Lambda_{N} \cap X} e^{2 \pi i x \xi} \neq 0
$$

where $\Lambda_{N}=[0, N]$.

Theorem 3.2 proves this conjecture under the hypothesis that $\hat{\mu}$ is a translation bounded measure. This is only of limited value, since as a rule $\hat{\mu}$ is not a measure if $X$ is not a lattice to start with. (Theorem 3.2 was, however, crucial for the proofs of Theorems 5.1 and 5.4.) More useful is Theorem 3.4: it shows that if the limit in (6.2) exists uniformly with respect to the position of $\Lambda_{N}$ and is equal to $m_{\xi}$, then $\hat{\gamma}(\{\xi\})=\left|m_{\xi}\right|^{2}$. This means that if the limit in (6.2) exists uniformly with respect to position, then the Fourier transform $\hat{\gamma}$ of the autocorrelation $\gamma$ has a point mass at $\xi$ of strength $\left|m_{\xi}\right|^{2}$ if $m_{\xi} \neq 0$, and no point mass at $\xi$ if $m_{\xi}=0$. Thus Theorem 3.4 proves a variation on a conjecture of Bombieri and Taylor. It is a variation in that it refers to $\hat{\gamma}$, the quantity that describes diffraction experiments, instead of to $\hat{\mu}$. In addition, it applies in arbitrary dimension.

Bombieri and Taylor have used their conjecture to obtain constraints on $X^{*}$ for sets $X$ defined by means of so-called primitive substitutions. Their method has been used for two-dimensional self-similar tilings in $[22,23]$. At least in some cases, Theorem 1 of [20] can be used to prove that the convergence in (6.2) is uniform with respect to the position of $\Lambda_{N}$ for those $\xi$ that satisfy the constraints $[28,27]$. Thus, by Theorem 3.4, the constraints are indeed constraints on the discrete part of $\hat{\gamma}$, i.e. on the discrete part of the diffraction spectrum.

The question whether the limit in (6.1) exists uniformly with respect to position for all $\xi$ is hard to answer in general because it amounts to the question whether all eigenfunctions of an associated dynamical system are continuous [45]. The associated dynamical system is obtained by letting $\mathbb{R}^{d}$ act on $X$ by translations. The set of all translates of $X$ can be closed in a topology of hard-sphere particle systems (see e.g. [29], Appendix B). This gives a compact metrizable space $\Omega$ with $\mathbb{R}^{d}$-action. (The minimality condition on $X$ mentioned above would be that the $\mathbb{R}^{d}$-orbit of every point of $\Omega$ is dense in $\Omega$.)

Another way of formulating Bombieri and Taylor's conjecture is that $\hat{\mu}$ should have a Dirac delta function at $\xi$ if $\widehat{\mu_{L}}(\xi)$ scales like $L$. This has been generalized: a scaling like $L^{\alpha}$, with $\frac{1}{2}<\alpha<1$, has been associated with a singular continuous part in $\hat{\gamma}[2,3,12,31]$; multifractal properties of $\hat{\gamma}$ have also been studied $[24,13]$. We are not aware of any rigorous result in this direction. (Note, however, that the existence of a unique autocorrelation $\gamma$ assures that the functions $L^{-1}\left|\widehat{\mu_{L}}\right|^{2}$ converge vaguely to $\hat{\gamma}$ as $L \rightarrow \infty$; the quantity $L^{-1}\left|\widehat{\mu_{L}}\right|^{2}$ is often called the "structure factor."

Aperiodic structures have been classified by the symmetries of their diffraction spectra by Mermin et al. [40,38,37].

Acknowledgements. It is a pleasure to thank the IHES for its hospitality. This work has been started in Groningen as part of my thesis [28] and was continued in Zürich and Bures-sur-Yvette. The work in Groningen has been supported by the Samenwerkingsverband Mathematische Fysica through the Stichting voor Fundamenteel Onderzoek der Materie. I would like to thank M. Duneau, A.C.D. van Enter, T.Q.T. Le, E.G.F. Thomas, and M. Winnink for discussions. 


\section{References}

1. Argabright, L., Gil de Lamadrid, J.: Fourier Analysis of Unbounded Measures on Locally Compact Abelian Groups. Providence, RI: American Mathematical Society, 1974. Number 145 of the Memoirs of the American Mathematical Society.

2. Aubry, S., Godrèche, C., Luck, J.M.: A structure intermediate between quasi-periodic and random. Europhys. Lett. 4, 639-643 (1987)

3. Aubry, S., Godrèche, C., Luck, J.M.: Scaling properties of a structure intermediate between quasiperiodic and random. J. Stat. Phys. 51, 1033-1074 (1988)

4. Aubry, S., Godrèche, C., Vallet, F.: Incommensurate structure with no average lattice: An example of an one-dimensional quasicrystal. J. Physique 48, 327-334 (1987)

5. Behrend, D., Radin, C.: Are there chaotic tilings? Commun. Math. Phys. 152, 215-219 (1993)

6. Bombieri, E., Taylor, J.E.: Which distributions diffract? An initial investigation. J. Phys. Colloque $47 \mathrm{C} 3,19-28$ (1986)

7. Bombieri, E., Taylor, J.E.: Quasicrystals, tilings, and algebraic number theory: Some preliminary connections. In: The Legacy of S. Kovalevskaya, pages 241-264. Providence, RI: American Mathematical Society, 1987. Volume 64 of Contemporary Mathematics.

8. de Bruijn, N.G.: Algebraic theory of Penrose's non-periodic tilings of the plane. I. Nederl. Akad. Wetensch. Proc. Ser. A, 84, 39-52 (1981) (= Indagationes Mathematicae 84, 39-52)

9. de Bruijn, N.G.: Algebraic theory of Penrose's non-periodic tilings of the plane. II. Nederl. Akad. Wetensch. Proc. Ser. A, 84, 53-66 (1981) (= Indagationes Mathematicae 84, 53-66)

10. de Bruijn, N.G.: Quasicrystals and their Fourier transform. Nederl. Akad. Wetensch. Proc. Ser. A, 89, 123-152 (1986) (= Indagationes Mathematicae 89, 123-152)

11. de Bruijn, N.G.: Modulated quasicrystals. Nederl. Akad. Wetensch. Proc. Ser. A, 90, 121-132 (1987) (= Indagationes Mathematicae 90, 121-132)

12. Cheng, Z., Savit, R.: Structure factor of substitutional sequences. J. Stat. Phys. 60, 383-393 (1990)

13. Cheng, Z., Savit, R.: Multifractal properties of the structure factor of a class of substitutional sequences. Phys. Rev. A 44, 6379-6385 (1991)

14. Cowley, J.M.: Diffraction Physics. Amsterdam: North-Holland, second edition, 1990

15. Dieudonné, J.: Éléments d'analyse, volume 2. Paris: Gauthier-Villars, 1969

16. Donoghue, W.F., Jr.: Distributions and Fourier Transforms. New York: Academic Press, 1969

17. Duneau, M.: Pavages, structures quasi-periodiques et modelisation des quasi-cristaux. In: Godrèche, C., editor, Du Cristal à l'Lamorphe, pages 157-197. Les Éditions de Physique, Les Ulis, France, 1988

18. Dworkin, S.: Spectral theory and X-ray diffraction. J. Math. Phys. 34, 2965-2967 (1993)

19. van Enter, A.C.D., Miękisz, J.: How should one define a (weak) crystal? J. Stat. Phys. 66, 1147-1153 (1992)

20. Geerse, C.P.M., Hof, A.: Lattice gas models on self-similar aperiodic tilings. Rev. Math. Phys 3, 163-221 (1991)

21. Gil de Lamadrid, J., Argabright, L.: Almost Periodic Measures. Providence, RI: American Mathematical Society, 1990. Number 428 of the Memoirs of the American Mathematical Society

22. Godrèche, C.: The sphinx: A limit-periodic tiling of the plane. J. Phys. A: Math. Gen. 22, L1163-L1166 (1989)

23. Godrèche, C., Lançon, F.: A simple example of a non-Pisot tiling with five-fold symmetry. J. Phys. I France 2, 207-220 (1992)

24. Godrèche, C., Luck, J.M.: Multifractal analysis in reciprocal space and the nature of the Fourier transform of self-similar structures. J. Phys. A: Math. Gen. 23, 3769-3797 (1990)

25. Guinier, A.: X-Ray Diffraction. W.H. Freeman and Company, 1963

26. Henley, C.L.: Quasicrystal order, its origins and its consequences: A survey of current models. Comments Cond. Mat. Phys. 13, 59-117 (1987)

27. Hof, A.: In preparation

28. Hof, A.: Quasicrystals, aperiodicity and lattice systems. PhD Thesis, University of Groningen, The Netherlands, 1992

29. Israel, R.B.: Convexity in the Theory of Lattice Gases. Princeton, NJ: Princeton University Press, 1979 
30. Katz, A., Duneau, M.: Quasiperiodic patterns and icosahedral symmetry. J. Physique 47, 181196 (1986)

31. Kolàr, M., Iochum, B., Raymond, L.: Structure factor of $1 \mathrm{~d}$ systems (superlattices) based on two-letter substitution rules I. $\delta$ (Bragg) peaks. J. Phy. A: Math. Gen. 26, 7343-7366 (1993)

32. Le, T.Q.T.: Local rules for quasiperiodic tilings. Preprint, Max-Planck Institut für Mathematik, Bonn

33. Le, T.Q.T., Piunikhin, S., Sadov, V.: Local rules for quasiperiodic tilings of quadratic 2-planes in $R^{4}$. Commun. Math. Phys. 150, 23-44 (1992)

34. Le, T.Q.T., Piunikhin, S., Sadov, V.: Geometry of quasicrystals. Russ. Math. Surv. 48, 41-102 (1993). In Russian

35. Levine, D., Steinhardt, P.J.: Quasicrystals: A new class of ordered structures. Phys. Rev. Lett. 53, 2477-2480 (1984)

36. Levine, D., Steinhardt, P.J.: Quasicrystals. I. definition and structure. Phys. Rev. B 34, 596-616 (1986)

37. Mermin, N.D.: Copernical crystallography. Phys. Rev. Lett. 68, 1172-1175 (1992)

38. Mermin, N.D.: The space groups of icosahedral quasicrystals and cubic, orthorhombic, monoclinic and triclinic crystals. Rev. Mod. Phys. 64, 3-49 (1992); Erratum: Rev. Mod. Phys. 64, 635 (1992)

39. Oguey, C., Duneau, M., Katz, A.: A geometrical approach of quasiperiodic tilings. Commun. Math. Phys. 118, 99-118 (1988)

40. Rabson, D.A., Mermin, N.D., Rohksar, D.S., Wright, D.C.: The space groups of axial crystals and quasicrystals. Rev. Mod. Phys. 63, 699-733 (1991)

41. Radin, C.: Global order from local sources. Bull. Am. Math. Soc. 25, 325-364 (1991)

42. Radin, C.: Symmetry of tilings of the plane. Bull. Am. Math. Soc. 29, 213-217 (1993)

43. Radin, C., Wolf, M.: Space tilings and local isomorphism. Geometrica Dedicata 42, 355-360 (1992)

44. Robinson, E.A., Jr.: The dynamical theory of tilings and quasicrystallography. Preprint, The George Washington University

45. Robinson, E.A., Jr.: On uniform convergence in the Wiener-Wintner theorem. J. Lond. Math. Soc. 49, 493-501 (1994)

46. Rudolph, D.J.: Rectangular tilings of $R^{n}$ and free $R^{n}$ actions. In: Alexander, J.C., editor, Dynamical Systems - Proceedings, University of Maryland 1986-87, pages 653-689. Berlin, Heidelberg, New York: Springer, 1988. Volume 1342 of Lecture Notes in Mathematics

47. Ruelle, D.: Statistical Mechanics. New York: W.A. Benjamin, Inc., 1969

48. Schwartz, L.: Théorie des Distributions. Paris: Hermann, new edition, 1966

49. Senechal, M., Taylor, J.: Quasicrystals: The view from Les Houches. Math. Intell. 12, 54-64 (1990)

50. Shechtman, D., Blech, I., Gratias, D., Cahn, J.W.: Metallic phase with longrange orientational order and no translational symmetry. Phys. Rev. Lett. 53, 1951-1953 (1984)

51. Solomyak, B.: Dynamics of self-similar tilings. Preprint, University of Washington

52. Wiener, N.: Generalized harmonic analysis. Acta Math. 55, 117-258 (1930). Reprinted as paper [30a] in Norbert Wiener: Collected Works, P. Masani, ed., The MIT Press

53. Wiener, N.: The Fourier Integral and Certain of its Applications. New York, N.Y.: Dover Publications, 1958

Communicated by J.L. Lebowitz 
